\title{
Spatial heterogeneity in epithelial to mesenchymal transition properties of circulating tumor cells associated with distant recurrence in pancreatic cancer patients
}

\author{
Xiu Dong ${ }^{1 \#}$, Yongsu Ma ${ }^{2 \#}$, Xudong Zhao ${ }^{2 \#}$, Xiaodong Tian ${ }^{2}$, Yulin Sun ${ }^{1}$, Yinmo Yang ${ }^{2}$, Xiaohang Zhao ${ }^{1}$ \\ ${ }^{1}$ State Key Laboratory of Molecular Oncology, National Cancer Center/National Clinical Research Center for Cancer/Cancer Hospital, Chinese \\ Academy of Medical Sciences \& Peking Union Medical College, Beijing, China; ${ }^{2}$ Department of General Surgery, Peking University First Hospital, \\ Beijing, China \\ Contributions: (I) Conception and design: Y Ma, X Zhao, Y Yang; (II) Administrative support: X Zhao, Y Yang; (III) Provision of study materials or \\ patients: X Zhao, Y Ma, Y Yang; (IV) Collection and assembly of data: X Dong, Y Ma, X Zhao, X Tian; (V) Data analysis and interpretation: All \\ authors; (VI) Manuscript writing: All authors; (VII) Final approval of manuscript: All authors. \\ \#These authors contributed equally to this work. \\ Correspondence to: Xiaohang Zhao. State Key Laboratory of Molecular Oncology, National Cancer Center/Cancer Hospital, Chinese Academy of \\ Medical Sciences, 17 Panjiayuan Nanli, Chaoyang District, Beijing, China. Email: zhaoxh@cicams.ac.cn; Yinmo Yang. Department of General \\ Surgery, Peking University First Hospital, 8 Xishiku Street, Xicheng District, Beijing, China. Email: yangyinmo@263.net.
}

Background: The spatial heterogeneity of epithelial to mesenchymal transition (EMT)-related circulating tumor cells (CTCs) within the circulatory system and its potential clinical relevance remain unclear in pancreatic cancer (PC) patients. We aimed to map the distribution of EMT-related CTCs along the spreading pathway and investigate the prognostic significance due to the potential spatial heterogeneity in the count and phenotypic properties of CTCs.

Methods: Both portal vein (PoV) and peripheral vein (PV) blood samples were collected from 39 PC patients. CTCs were isolated by using a CD45 negative enrichment method, and EMT-related phenotypes in CTCs were analyzed by 4-channel immunofluorescence. The correlations of CTCs with patient characteristics and recurrence-free survival (RFS) were analyzed.

Results: Both the number \{median CTC total count, 10 [6-16] in PoV vs. 5 [1-7] in PV per mL, $\mathrm{P}<0.0001$ \} and EMT status of CTCs [median mesenchymal CTC (M-CTC) percentage, 0.33 (0.13-0.52) in PoV vs. $0.2(0-0.4)$ in $\mathrm{PV}, \mathrm{P}=0.0211$ ] showed significant spatial heterogeneity during dissemination from the PoV to the PV. Univariate analysis adjusting for patient age and sex revealed that CTC total count and M-CTC percentage in PoV samples could be risk factors for RFS in PC patients ( $\mathrm{P}=0.003$ and $\mathrm{P}=0.001$, respectively), and ROC curve analysis found that both of these factors had good performance in distinguishing patients with early distant recurrence (within 6 months), with the optimal cut-off values of 14 cells/mL (AUROC $=0.893$, sensitivity $=0.857$, specificity $=0.813, \mathrm{P}=0.001)$ and 0.545 (AUROC $=0.795$, sensitivity $=0.714$, specificity $=0.906, \mathrm{P}=0.016$ ), respectively.

Conclusions: Multivascular assessment of EMT-related CTCs suggested profound dynamic alterations in total count and phenotypes during dissemination, and the spatial heterogeneity of CTCs in circulation could help establish novel prognosis markers in PC patients.

Keywords: Circulating tumor cells (CTCs); epithelial to mesenchymal transition (EMT); pancreatic cancer (PC); prognosis; spatial heterogeneity

Submitted Jan 15, 2020. Accepted for publication May 13, 2020.

doi: $10.21037 / \mathrm{atm}-20-782$

View this article at: http://dx.doi.org/10.21037/atm-20-782 


\section{Introduction}

Pancreatic cancer (PC) is among the most lethal malignances worldwide, highlighted by the almost same rates of disease incidence and cancer-related mortality $(1,2)$. Currently, curative strategy for PC patients is still radical surgery (3), but only $10-20 \%$ of patients have tumors that are regarded as resectable at initial diagnosis (4). Furthermore, even after a potential radical resection, most patients will ultimately experience recurrence, especially liver metastasis $(5,6)$, with approximately $20 \%$ recurrence within the first six months (termed early recurrence) $(6,7)$. Therefore, there is an urgent need to identify appropriate biomarkers to better stratify PC patients for surgery.

Circulating tumor cells (CTCs) are cells shed from primary tumors that migrate into the circulation and are considered key to the development of distant metastases $(8,9)$. Previous studies have shown that epithelial to mesenchymal transition (EMT) contributes to this dissemination process, and EMT is associated with phenotypic conversion that results in the downregulation of epithelial characteristics and the upregulation of mesenchymal features in CTCs $(8,10-12)$. CTCs can be classified into three subgroups: epithelial (E-CTC), mesenchymal (M-CTC), and epithelial/mesenchymal hybrids (E/M-CTC). It is hypothesized that each subpopulation of CTCs that reaches the circulation might have distinct properties (12-14).

The quantification and subtyping of CTCs in the peripheral blood could be a potential real-time and noninvasive approach to predict tumor recurrence and prognosis $(14,15)$. Because CTCs in PC patients have initial access to the portal circulation, where they can directly reach the liver, comparative analysis of CTCs both in the portal and systemic circulation could help better understand the role of CTCs in metastases and predict prognosis (16). Tien et al. compared CTC counts between portal vein $(\mathrm{PoV})$ and peripheral vein $(\mathrm{PV})$ blood samples collected during pancreaticoduodenectomy in 41 patients, and found that CTCs were detected in $58.5 \%$ of portal and $39 \%$ of PV samples from patients, and a high CTC count in portal blood was a significant predictor for early postoperative liver metastases (17). However, the majority of such studies focused on CTC counts only, without examining the EMT status of CTCs.

Many different methods of CTC isolation have been developed based on the physical properties, such as size exclusion (18) of tumor cells, the positive selection of tumor cell surface markers (19), or the negative removal of white blood cells (20). However, there has not been any unified method so far due to the complexity of CTCs (21). To analyze the distinct EMT status of CTCs, density gradient centrifugation for blood sample pretreatment and CD45 white blood cell depletion for CTC negative enrichment were applied in our study.

Therefore, in this study, we aimed to identify CTCs in PoV and PV samples from 39 PC patients and explore possible dynamic changes in CTCs in circulation. Furthermore, we analyzed the relationship between CTC characteristics in PoV samples and the clinicopathologic data of PC patients, particularly those parameters indicating early recurrence in patients who had undergone resection.

\section{Methods}

\section{Patients and characteristics}

This study was carried out with the approval of the Institutional Review Board at the Peking University First Hospital (ID: 2018 keyan-15), and in compliance with the guidelines of the Declaration of Helsinki.

A total of 39 consecutive PC patients who were treated with curative surgical resection combined with adjuvant therapy at the Peking University First Hospital between March 2018 and April 2019 were recruited, and informed consent was obtained from all the patients. PV blood (at least $6 \mathrm{~mL}$ ) was collected prior to the surgery, and PoV blood (also at least $6 \mathrm{~mL}$ ) was then obtained by direct intraoperative venipuncture before manipulating the tumor during pancreaticoduodenectomy or distal pancreatectomy.

Patients were followed with standard postoperative visits every 3 months to monitor tumor recurrence. Follow-up was terminated on October 30, 2019, and recurrence-free survival (RFS) was defined as the time interval between the date of operation and the date of recurrence or the endpoint of follow-up.

\section{Isolation and identification of CTCs}

CTCs were isolated by the CD45 negative enrichment method. First, peripheral or portal blood mononuclear cells were collected by density gradient centrifugation using Histopaque-1077 (Sigma-Aldrich, St. Louis, MO, USA). Next, mononuclear cells were mixed with microbeads (Miltenyi Biotec, Bergisch Gladbach, Germany) conjugated to monoclonal anti-human CD45 antibodies and incubated 
for $15 \mathrm{~min}$ at $4{ }^{\circ} \mathrm{C}$. Subsequently, the cells and microbeads were loaded onto a LS column for magnetic separation (Miltenyi Biotec). The suspensions of unlabeled CD45cells were collected and spread on a glass slide for immunofluorescence staining. In brief, cells were fixed and then incubated with anti-CK-FITC (Miltenyi Biotec, catalog: 130-080-101) (1:100 diluted in 2\% BSA), antiVimentin-Alexa Fluor 647 (Cell Signaling Technology, Danvers, MA, USA) (1:200 diluted in 2\% BSA) or antiCD45-PE (Miltenyi Biotec) (1:300 diluted in 2\% BSA) antibodies for $1 \mathrm{~h}$ at room temperature. The slide was washed three times with PBS. Cells were mounted with the nuclear dye 4',6-diamidino-2-phenylindole (DAPI) (Sigma-Aldrich) and ProLong Gold antifade mountant (Invitrogen, Carlsbad, California, USA). CTC slides were loaded on a Leica SP8 laser scanning confocal microscope (Leica Microsystems, Heidelberg, Baden-Wurttemberg, Germany), and cells were observed to acquire the entire fluorescence signal of each multicolor channel. The areas with cells on the slides were marked and subjected to automated full $\mathrm{X}-\mathrm{Y}-\mathrm{Z}$ scanning. Then, we counted CTCs manually via scanned multiple fluorescent images. The formula to calculate the CTC number of each sample was the CTC count of each slide divided by the volume of blood sample. The identification criteria for positive CTCs in this study were $\mathrm{DAPI}^{+} / \mathrm{CD}^{-} 5^{-} / \mathrm{CK}^{+}$and/or Vimentin ${ }^{+}$cells.

\section{Statistical analysis}

All statistical analyses were performed using SPSS 25.0 (IBM Corp., Armonk, NY, USA) and Prism 7.0 (GraphPad, La Jolla, CA, USA), and a two-tailed $\mathrm{P}$ value $\leq 0.05$ was considered significant. Data are presented as frequencies and percentages for categorical variables and as the mean values with ranges for continuous variables. The MannWhitney $U$ test was used to compare the data not normally distributed. Differences in RFS between patient groups were tested using the log-rank test, and hazard ratios were estimated from Cox proportional hazards models that adjusted for age and sex. A receiver operating characteristic (ROC) curve was established to determine the best cut-off value and evaluate the prognostic value of CTCs.

\section{Results}

\section{CTCs exhibit profound dynamic changes during dissemination}

CTCs and a few residual CD $45^{+}$white blood cells were observed in the multiple fluorescent images (Figure S1). CTCs were divided into three subtypes based on staining: (I) epithelial CTCs (E-CTCs), $\mathrm{CK}^{+} /$Vimentin $^{-} / \mathrm{CD}^{2} 5^{-} /$ DAPI $^{+}$; (II) epithelial/ mesenchymal hybrid CTCs (E/ M-CTCs), $\mathrm{CK}^{+} / \mathrm{Vimentin} / \mathrm{CD} 45^{-} / \mathrm{DAPI}^{+}$; and (III) mesenchymal CTCs (M-CTCs), $\mathrm{CK}^{-} / \mathrm{Vimentin}^{+} / \mathrm{CD} 45^{-} /$ $\mathrm{DAPI}^{+}$(Figure $1 A$ ). Among all 39 patients, 36 patients (92\%) had detectable CTCs in portal blood, while 29 patients (74\%) presented positive CTCs in peripheral blood. The CTC total count in portal blood was significantly higher than that in peripheral blood \{median CTC total count, 10 [6-16] in PoV vs. 5 [1-7] in PV per $\mathrm{mL}, \mathrm{P}<0.0001\}$ (Figure 1 B). Furthermore, we analyzed CTC subgroups and found that only the percentage of M-CTCs but not that of E-CTCs or E/M-CTCs was significantly different between portal blood and peripheral blood [median M-CTC percentage, 0.33 (0.13-0.52) in $\mathrm{PoV}$ vs. $0.2(0-0.4)$ in $\mathrm{PV}, \mathrm{P}=0.0211$ ] (Figure 1C). These results suggest profound dynamic changes in the enumeration and EMT status of CTCs from the PoV to the peripheral circulation.

\section{Correlation between clinical parameters and CTC count or M-CTC percentage in portal blood}

The CTC count in portal blood was positively correlated with the tumor microvascular invasion state $(\mathrm{P}=0.022)$, but not with other clinicopathological features. Meanwhile, the percentage of M-CTCs in portal blood was only significantly correlated with tumor size $(\mathrm{P}=0.018)$, but not with other clinicopathological features (Table 1).

\section{CTC count and M-CTC percentage in portal blood as prognostic biomarkers in $P C$ patients}

After a median follow-up of 41 weeks, 17 patients experienced recurrence and all of them had distant 

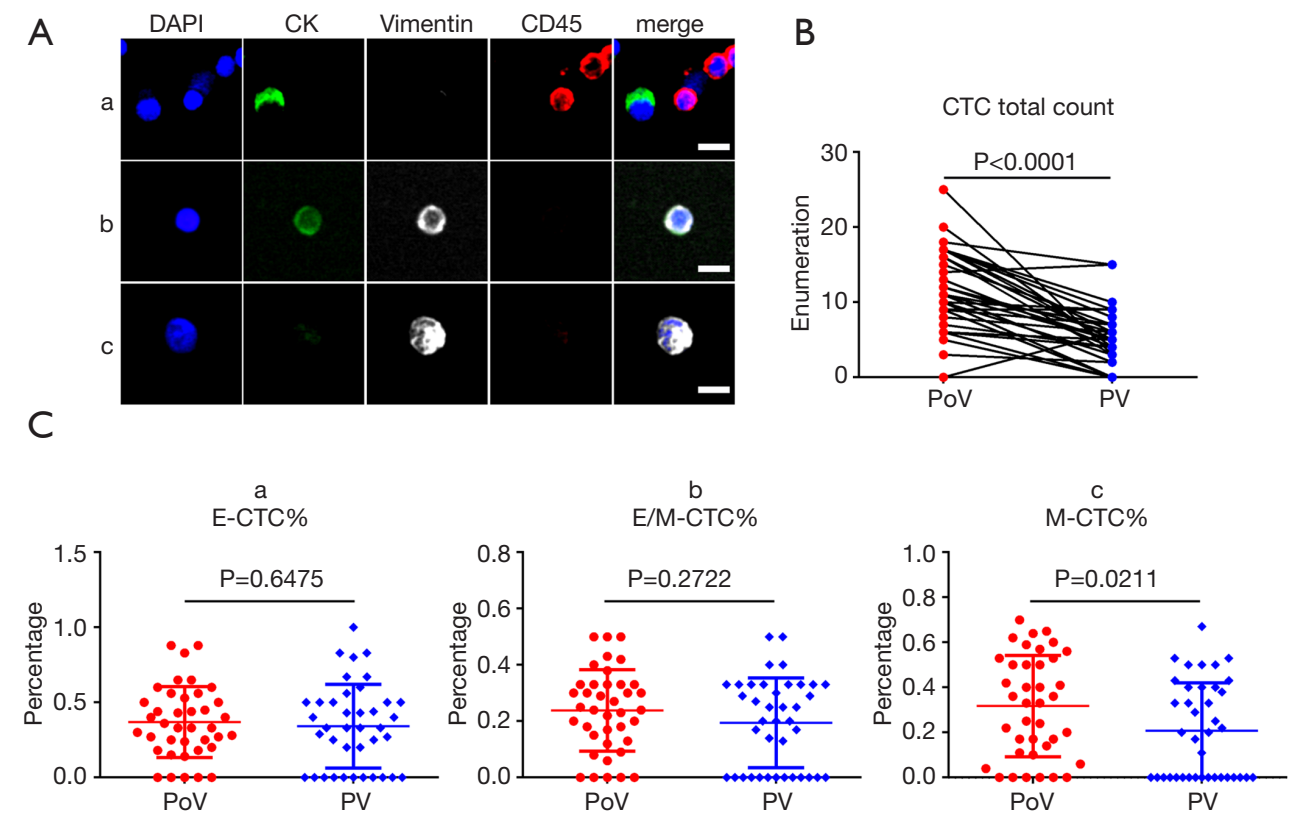

Figure 1 CTCs exhibit profound spatial heterogeneity during dissemination. (A) Immunofluorescence staining of isolated CTCs, (a) epithelial CTCs, E-CTCs are $\mathrm{CK}^{+} / \mathrm{Vimentin}^{-} / \mathrm{CD}^{-} 5^{-} / \mathrm{DAPI}^{+}$; (b) epithelial/mesenchymal hybrid CTCs, E/M-CTCs are $\mathrm{CK}^{+} / \mathrm{Vimentin}^{+} /$ CD45 $/ \mathrm{DAPI}^{+}$; and (c) mesenchymal CTCs, M-CTCs are $\mathrm{CK}^{-} /$Vimentin ${ }^{+} / \mathrm{CD}^{2} 5^{-} / \mathrm{DAPI}^{+}$. Scale bar: $10 \mu \mathrm{m}$. (B) The CTC total count in portal blood was significantly higher than that in peripheral blood \{median, 10 [6-16] in $\mathrm{PoV}$ vs. 5 [1-7] in $\mathrm{PV}$ per $\mathrm{mL}$, $\mathrm{P}<0.0001$ \}. (C) The percentage of M-CTCs (c) but not that of E-CTCs (a) or E/M-CTCs (b) showed a significant difference between portal blood and peripheral blood [median, 0.33 (0.13-0.52) in PoV vs. 0.2 (0-0.4) in $\mathrm{PV}, \mathrm{P}=0.0211$ ].

metastases to the liver or lung. The optimal cut-off values of CTCs in both portal and peripheral blood for estimating survival were explored by ROC analysis. We found that both CTC total count and M-CTC percentage in portal blood had good performance, with a cut-off value of $10 / \mathrm{mL}$ for count (sensitivity $=0.941$, specificity $=0.682, \mathrm{P}<0.001$ ) and 0.405 for M-CTC percentage (sensitivity $=0.765$, specificity $=0.909, \mathrm{P}<0.001$ ), respectively.

Based on the cut-off values, univariate analysis adjusting for age and sex showed that the prognostic indicators included tumor size $(\mathrm{P}=0.039)$, lymph node status $(\mathrm{P}=0.008)$, AJCC stage $(\mathrm{P}=0.017)$, microvascular invasion $(\mathrm{P}=0.019)$, CTC total count in $\mathrm{PoV}(\mathrm{P}=0.003)$ and $\mathrm{M}-\mathrm{CTC}$ percentage $(\mathrm{P}=0.001$ in $\mathrm{PoV}$ and $\mathrm{P}=0.003$ in $\mathrm{PV}$ ) (Table 2). KaplanMeier analysis showed that CTC total count (31 weeks vs. undefined, HR $=18.95,95 \%$ CI: 7.297-49.19, log-rank test $\mathrm{P}<0.0001)$ and $\mathrm{M}-\mathrm{CTC}$ ratio $(26$ weeks $v s$. undefined, $\mathrm{HR}=8.576,95 \% \mathrm{CI}: 2.957-24.87, \log$-rank test $\mathrm{P}<0.0001$ ) in portal blood were significantly associated with tumor recurrence (Figure 2).

Moreover, 7 patients (18\%) had early recurrence (within 6 months), and all of them had intrahepatic metastasis within six months. As CTCs in the PoV may potentially be the direct cause of liver micrometastases, we performed ROC analysis and found that both the CTC total count and M-CTC percentage in portal blood had good performance in distinguishing patients with early distant recurrence, with optimal cut-off values of $14 / \mathrm{mL}$ (AUROC $=0.893$, sensitivity $=0.857$, specificity $=0.813, \mathrm{P}=0.001)$ and 0.545 (AUROC $=0.795$, sensitivity $=0.714$, specificity $=0.906$, $\mathrm{P}=0.016)$, respectively.

\section{Discussion}

Given the poor outcome among PC patients who have undergone resection, current studies have focused on reliable biomarkers that can aid in stratifying a patient's risk for distant recurrence (22). CTCs are deemed precursors of metastasis, and previous studies have revealed that the transport of tumor cells across the bloodstream is involved in metastases (23). Theoretically, tracking and analyzing CTCs in the circulation from the PoV to the PV could help monitor cancer metastasis and allow better risk stratification of cancer patients for appropriate therapies $(16,23)$. 
Table 1 Correlation between clinical parameters and CTC count or M-CTC percentage in portal blood

\begin{tabular}{|c|c|c|c|c|c|}
\hline Characteristics & $\mathrm{n}$ & \multicolumn{2}{|c|}{ СТС } & \multicolumn{2}{|c|}{ М-СТС } \\
\hline All patients & 39 & & & & \\
\hline Sex & & & 0.424 & & 0.201 \\
\hline Male & 22 & $10(6-15.3)$ & & $0.38(0.17-0.58)$ & \\
\hline Age (years) & & & 0.235 & & 0.072 \\
\hline$>65$ & 19 & $11[9-16]$ & & $0.41(0.21-0.55)$ & \\
\hline$\leq 65$ & 20 & $9.5(5.8-12.3)$ & & $0.21(0-0.41)$ & \\
\hline Tumor location & & & 0.878 & & 0.212 \\
\hline CA19-9 level (U/mL) & & & 0.322 & & 0.825 \\
\hline$>37$ & 30 & $11(8.3-15.8)$ & & $0.35(0.13-0.50)$ & \\
\hline$\leq 37$ & 9 & 7 [5-12] & & $0.17(0.14-0.56)$ & \\
\hline Differentiation & & & 0.309 & & 0.892 \\
\hline Poor & 15 & $12(7.5-16.5)$ & & $0.33(0.14-0.46)$ & \\
\hline Good/moderate & 24 & $10(6-12.3)$ & & $0.36(0.13-0.53)$ & \\
\hline Tumor size (mm) & & & 0.062 & & 0.018 \\
\hline$>40$ & 15 & $13(9.5-16)$ & & $0.50(0.25-0.58)$ & \\
\hline IIB-III & 20 & $11.5(8.8-16.3)$ & & $0.39(0.11-0.54)$ & \\
\hline$I-I I A$ & 19 & $9(5.5-12)$ & & $0.33(0.17-0.45)$ & \\
\hline Perineural invasion & & & 0.403 & & 0.380 \\
\hline Positive & 36 & $10(6-15.3)$ & & $0.29(0.11-0.51)$ & \\
\hline Negative & 3 & $12(10.5-15)$ & & $0.42(0.38-0.49)$ & \\
\hline Microvascular invasion & & & 0.022 & & 0.370 \\
\hline Positive & 15 & $12(10-16.5)$ & & $0.41(0.11-0.54)$ & \\
\hline Negative & 24 & $8.5(5-12.3)$ & & $0.25(0.16-0.51)$ & \\
\hline
\end{tabular}

AJCC, American Joint Committee on Cancer; CA19-9, carbohydrate antigen 19-9; CTC, circulating tumor cell; M-CTC, CTC with a mesenchymal phenotype. 
Table 2 Univariate analysis of risk factors associated with RFS, adjusted for patient age and sex

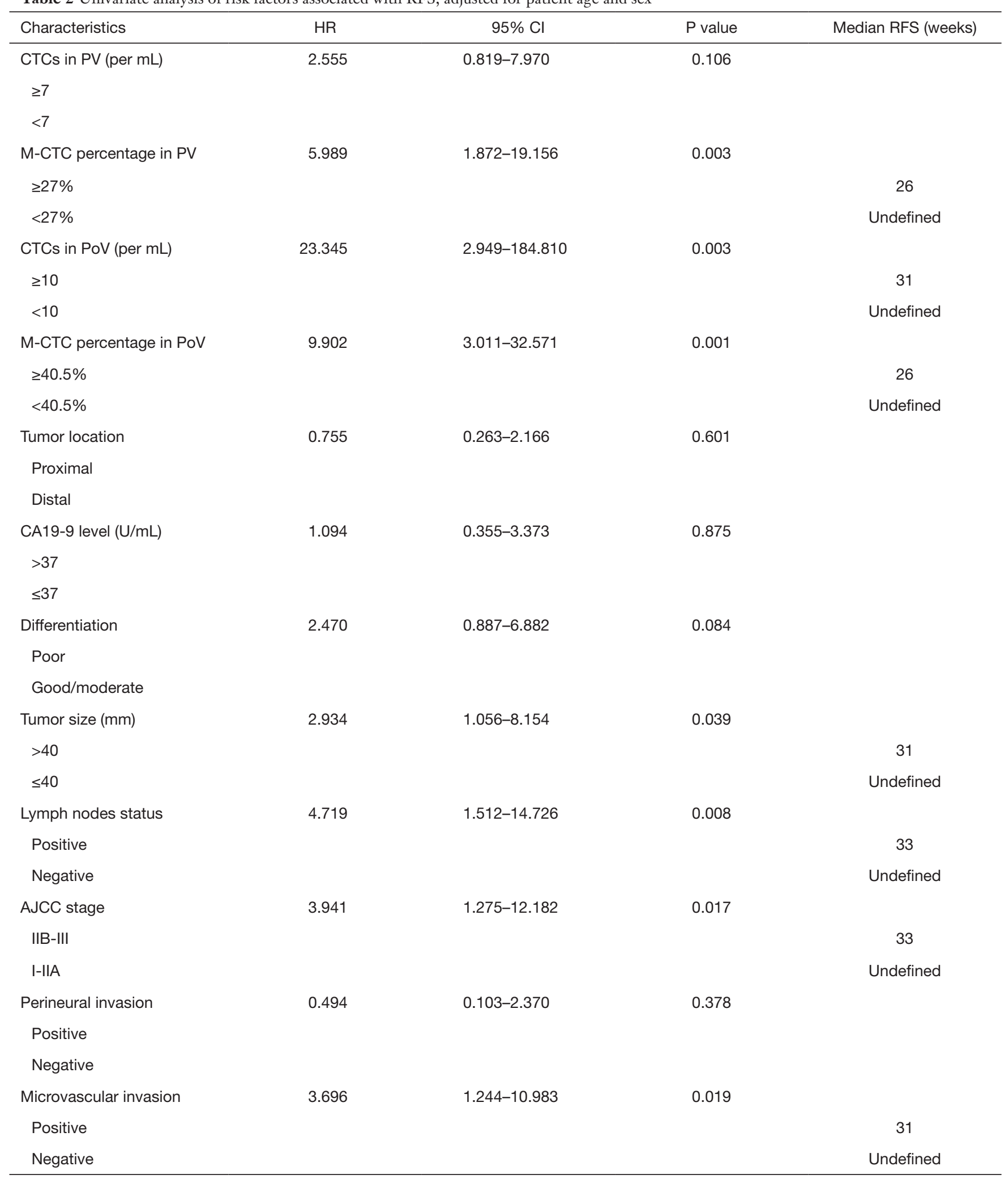

RFS, recurrence free survival; CTCs, circulating tumor cells; M-CTC, CTCs with a mesenchymal phenotype; PoV, portal vein; PV, peripheral vein; AJCC, American Joint Committee on Cancer; CA19-9, carbohydrate antigen 19-9. 

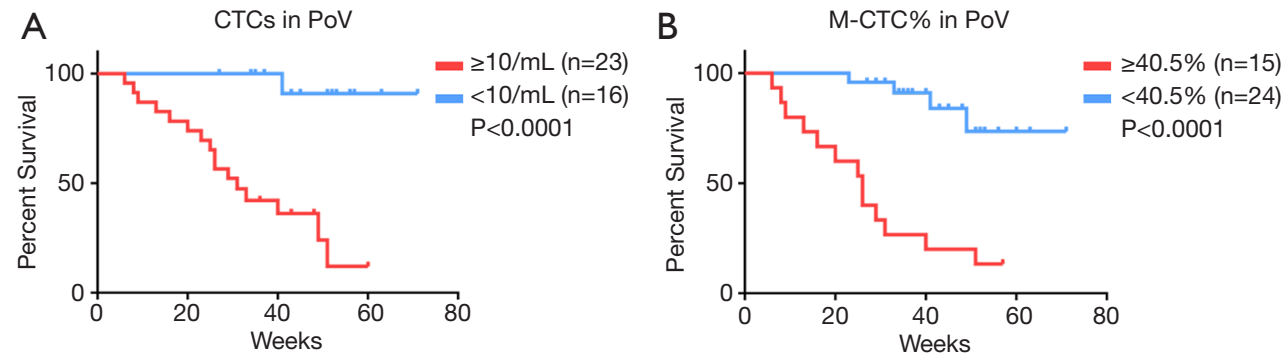

Figure 2 CTC total count and M-CTC percentage in portal blood as risk factors for distant recurrence by Kaplan-Meier analysis. (A) Patients with higher CTC total counts in PoV presented with inferior RFS than those with lower CTC total counts in PoV (31 weeks vs. undefined, $\mathrm{HR}=18.95$, 95\% CI: 7.297-49.19, log-rank test $\mathrm{P}<0.0001$ ). (B) The M-CTC percentage in portal blood was significantly associated with tumor recurrence (26 weeks vs. undefined, HR =8.576, 95\% CI: 2.957-24.87, log-rank test $\mathrm{P}<0.0001$ ). CTCs, circulating tumor cells; M-CTC, CTCs with a mesenchymal phenotype; PoV, portal vein.

To our knowledge, this is the first comprehensive study to characterize the EMT features of CTCs across the spreading route in localized PC patients and evaluate the potential clinical significance of CTCs. We found that CTC total count and the mesenchymal subphenotype of CTCs in portal blood could be high risk factors for postoperative distant metastases, especially early recurrence (within 6 months).

Pairwise analysis of CTC counts between portal and peripheral blood samples showed that the CTC total count in portal blood was significantly higher than that in peripheral blood, consistent with the results of previous studies $(17,24,25)$. This may be due to the potential filtration effect of liver-lung compartments that could enhance the retention of CTCs and prevent the release of CTCs into peripheral circulation (25). In addition, portal venous circulation constitutes an immunosuppressive microenvironment that could favor CTC survival and spread to the liver (26). However, one patient in our cohort had the opposite result, with a higher CTC count in peripheral blood (14/mL in PoV vs. $15 / \mathrm{mL}$ in PV). The underlying reason might be that CTCs in the periphery are released not only from the primary tumor but also from tumors with distant metastasis in the liver or lung. This speculation was confirmed by the follow-up in this patient, who had a significantly decreased DFS (liver metastasis within 2 months) after surgery.

In agreement with previous studies $(8,27)$, our results showed that CTCs could undergo EMT to disseminate from primary tumors and migrate into blood circulation. Rhim et al. found that circulating epithelial PC cells could exhibit mesenchymal phenotypes with invasive and tumorinitiating properties during natural evolution in vivo (8). The presence of M-CTCs has been linked to aggressive tumors, and patients with malignant characteristics such as advanced AJCC stage or distant metastases had a much higher ratio of M-CTCs than patients with more benign characteristics for example early AJCC stage (13-15). The dynamic changes in the EMT status of CTCs from the portal blood to the peripheral blood suggest that the acquisition of mesenchymal characteristics by CTCs may facilitate liver-lung tissue retention in liver cancer (28). Consistent with this speculation, our present study showed that the percentage of M-CTCs in peripheral blood was significantly lower than that in portal blood. Moreover, we found s correlation between a higher M-CTC percentage in portal blood and significantly decreased RFS in PC patients who had undergone resection.

In particular, PC patients have diverse patterns and timing of recurrence, with some presenting with liver metastases alone, and others coupled with liver and lung metastases synchronously or heterochronously $(5,29)$. Moreover, the spatial phenotypic disparities and characteristics of CTCs could determine the preferential site of metastasis. While hybrid CTCs (E/M-CTCs) might be a critical factor for intrahepatic metastasis, M-CTCs may predict extrahepatic metastasis. In addition, smaller CTCs might have the capability to pass through the liver compartments to the systemic circulation, but the larger CTCs tend to be detained in liver tissue in hepatocellular carcinoma patients $(28,30)$. These findings pave the way for understanding diverse PC metastatic patterns by analyzing CTCs from the different locations along their migration route.

Next, we explored the potential factors associated with the M-CTC percentage. Cells that have undergone EMT have enhanced capacities to survive and maintain aggressive properties $(10,12)$. We found that only the tumor size had 
a significant impact on the M-CTC percentage. Thus, we speculated that tumor size may reflect tumor aggressiveness due to the higher M-CTC percentage in portal blood because increased tumor size is associated with poor prognosis.

The limitations of this study are the small cohort size, short follow-up time, and data from a single center. Moreover, although we showed spatial heterogeneity of CTCs during their spreading, the potential molecular mechanism is still unclear. However, with the aid of singlecell sequencing of CTCs, we may be able to determine the underlying mechanisms and facilitate the prediction of distant metastasis patterns (31).

\section{Conclusions}

The EMT properties of CTCs showed significant changes during circulation from the $\mathrm{PoV}$ to the $\mathrm{PV}$ in $\mathrm{PC}$ patients. Multivascular detection and subphenotyping of CTCs could help establish novel biomarkers for disseminated metastasis and poor prognosis, while further large multicenter studies are needed to confirm our conclusions.

\section{Acknowledgments}

The authors thank Yan Zhuang, Zhengfei Zhou, Hongqiao Gao, Xuehai Xie, Yiran Chen, Shouge Zang and Jing Zhu from Peking University First Hospital for the collection and preparation of the samples.

Funding: This work was supported by grants from the National Key R \& D Program of China (No. 2018YFC1313101, 2017ZX10203205-003-001 and 2016YGC0901403 to Xiaohang Zhao), the National Natural Science Foundation of China (No. 81872033 and 81871954 to $\mathrm{XZ}$ and $\mathrm{YY}$ ), CAMS Innovation Fund for Medical Sciences (No. 2019-I2M-1-003, 2016-I2M-1-001 and 2017I2M-3-005 to XZ and YS) and the Youth Clinical Research Project of Peking University First Hospital (No. 2019CR18 to YM).

\section{Footnote}

Peer Review File: Available at http://dx.doi.org/10.21037/ atm-20-782

Conflicts of Interest: All authors have completed the ICMJE uniform disclosure form (available at http://dx.doi. org/10.21037/atm-20-782). The authors have no conflicts of interest to declare.
Ethical Statement: The authors are accountable for all aspects of the work in ensuring that questions related to the accuracy or integrity of any part of the work are appropriately investigated and resolved. This study was carried out with the approval of the Institutional Review Board at the Peking University First Hospital (ID: 2018 keyan-15) and in compliance with the guidelines of the Declaration of Helsinki. Written informed consent was obtained from all participants.

Open Access Statement: This is an Open Access article distributed in accordance with the Creative Commons Attribution-NonCommercial-NoDerivs 4.0 International License (CC BY-NC-ND 4.0), which permits the noncommercial replication and distribution of the article with the strict proviso that no changes or edits are made and the original work is properly cited (including links to both the formal publication through the relevant DOI and the license). See: https://creativecommons.org/licenses/by-nc-nd/4.0/.

\section{References}

1. Kamisawa T, Wood LD, Itoi T, et al. Pancreatic cancer. Lancet 2016;388:73-85.

2. Siegel RL, Miller KD, Jemal A. Cancer statistics, 2019. CA Cancer J Clin 2019;69:7-34.

3. Tempero MA, Malafa MP, Chiorean EG, et al. Pancreatic Adenocarcinoma, Version 1.2019. J Natl Compr Canc Netw 2019;17:202-10.

4. Piatek M, Kusnierz K, Bienkowski M, et al. Primarily resectable pancreatic adenocarcinoma - to operate or to refer the patient to an oncologist? Crit Rev Oncol Hematol 2019;135:95-102.

5. Jones RP, Psarelli EE, Jackson R, et al. Patterns of Recurrence After Resection of Pancreatic Ductal Adenocarcinoma: A Secondary Analysis of the ESPAC-4 Randomized Adjuvant Chemotherapy Trial. JAMA Surg 2019;154:1038-48.

6. Groot VP, Rezaee N, Wu W, et al. Patterns, Timing, and Predictors of Recurrence Following Pancreatectomy for Pancreatic Ductal Adenocarcinoma. Ann Surg 2018;267:936-45.

7. Labori KJ, Katz MH, Tzeng CW, et al. Impact of early disease progression and surgical complications on adjuvant chemotherapy completion rates and survival in patients undergoing the surgery first approach for resectable pancreatic ductal adenocarcinoma - A population-based cohort study. Acta Oncol 2016;55:265-77. 
8. Rhim AD, Mirek ET, Aiello NM, et al. EMT and dissemination precede pancreatic tumor formation. Cell 2012;148:349-61.

9. Plaks V, Koopman CD, Werb Z. Cancer. Circulating tumor cells. Science 2013;341:1186-8.

10. Aiello NM, Maddipati R, Norgard RJ, et al. EMT Subtype Influences Epithelial Plasticity and Mode of Cell Migration. Dev Cell 2018;45:681-95.e4.

11. Yu M, Bardia A, Wittner BS, et al. Circulating breast tumor cells exhibit dynamic changes in epithelial and mesenchymal composition. Science 2013;339:580-4.

12. Pastushenko I, Brisebarre A, Sifrim A, et al. Identification of the tumour transition states occurring during EMT. Nature 2018;556:463-8.

13. Zhao XH, Wang ZR, Chen CL, et al. Molecular detection of epithelial-mesenchymal transition markers in circulating tumor cells from pancreatic cancer patients: Potential role in clinical practice. World J Gastroenterol 2019;25:138-50.

14. Wei T, Zhang X, Zhang Q, et al. Vimentin-positive circulating tumor cells as a biomarker for diagnosis and treatment monitoring in patients with pancreatic cancer. Cancer Lett 2019;452:237-43.

15. Poruk KE, Valero V 3rd, Saunders T, et al. Circulating Tumor Cell Phenotype Predicts Recurrence and Survival in Pancreatic Adenocarcinoma. Ann Surg 2016;264:1073-81.

16. Buscail E, Chiche L, Laurent C, et al. Tumor-proximal liquid biopsy to improve diagnostic and prognostic performances of circulating tumor cells. Mol Oncol 2019;13:1811-26.

17. Tien YW, Kuo HC, Ho BI, et al. A High Circulating Tumor Cell Count in Portal Vein Predicts Liver Metastasis From Periampullary or Pancreatic Cancer: A High Portal Venous CTC Count Predicts Liver Metastases. Medicine (Baltimore) 2016;95:e3407.

18. Vona G, Sabile A, Louha M, et al. Isolation by size of epithelial tumor cells: a new method for the immunomorphological and molecular characterization of circulatingtumor cells. Am J Pathol 2000;156:57-63.

19. Allard WJ, Matera J, Miller MC, et al. Tumor cells circulate in the peripheral blood of all major carcinomas but not in healthy subjects or patients with nonmalignant diseases. Clin Cancer Res 2004;10:6897-904.

20. Ogle LF, Orr JG, Willoughby CE, et al. Imagestream detection and characterisation of circulating tumour cells - A liquid biopsy for hepatocellular carcinoma? J Hepatol 2016;65:305-13.
21. Harouaka R, Kang Z, Zheng SY, et al. Circulating tumor cells: advances in isolation and analysis, and challenges for clinical applications. Pharmacol Ther 2014;141:209-21.

22. Kleeff J, Korc M, Apte M, et al. Pancreatic cancer. Nat Rev Dis Primers 2016;2:16022.

23. Lambert AW, Pattabiraman DR, Weinberg RA. Emerging Biological Principles of Metastasis. Cell 2017;168:670-91.

24. Liu X, Li C, Li J, et al. Detection of CTCs in portal vein was associated with intrahepatic metastases and prognosis in patients with advanced pancreatic cancer. J Cancer 2018;9:2038-45.

25. Vilhav C, Engstrom C, Naredi P, et al. Fractional uptake of circulating tumor cells into liver-lung compartments during curative resection of periampullary cancer. Oncol Lett 2018;16:6331-8.

26. Arnoletti JP, Zhu X, Almodovar AJ, et al. Portal Venous Blood Circulation Supports Immunosuppressive Environment and Pancreatic Cancer Circulating Tumor Cell Activation. Pancreas 2017;46:116-23.

27. Celia-Terrassa T, Meca-Cortes O, Mateo F, et al. Epithelial-mesenchymal transition can suppress major attributes of human epithelial tumor-initiating cells. J Clin Invest 2012;122:1849-68.

28. Sun YF, Guo W, Xu Y, et al. Circulating Tumor Cells from Different Vascular Sites Exhibit Spatial Heterogeneity in Epithelial and Mesenchymal Composition and Distinct Clinical Significance in Hepatocellular Carcinoma. Clin Cancer Res 2018;24:547-59.

29. Groot VP, Gemenetzis G, Blair AB, et al. Implications of the Pattern of Disease Recurrence on Survival Following Pancreatectomy for Pancreatic Ductal Adenocarcinoma. Ann Surg Oncol 2018;25:2475-83.

30. Liu YK, Hu BS, Li ZL, et al. An improved strategy to detect the epithelial-mesenchymal transition process in circulating tumor cells in hepatocellular carcinoma patients. Hepatol Int 2016;10:640-6.

31. Su Z, Wang Z, Ni X, et al. Inferring the Evolution and Progression of Small-Cell Lung Cancer by Single-Cell Sequencing of Circulating Tumor Cells. Clin Cancer Res 2019;25:5049-60.

Cite this article as: Dong $\mathrm{X}, \mathrm{Ma} \mathrm{Y}$, Zhao X, Tian X, Sun $\mathrm{Y}$, Yang Y, Zhao X. Spatial heterogeneity in epithelial to mesenchymal transition properties of circulating tumor cells associated with distant recurrence in pancreatic cancer patients. Ann Transl Med 2020;8(11):676. doi: 10.21037/atm-20-782 

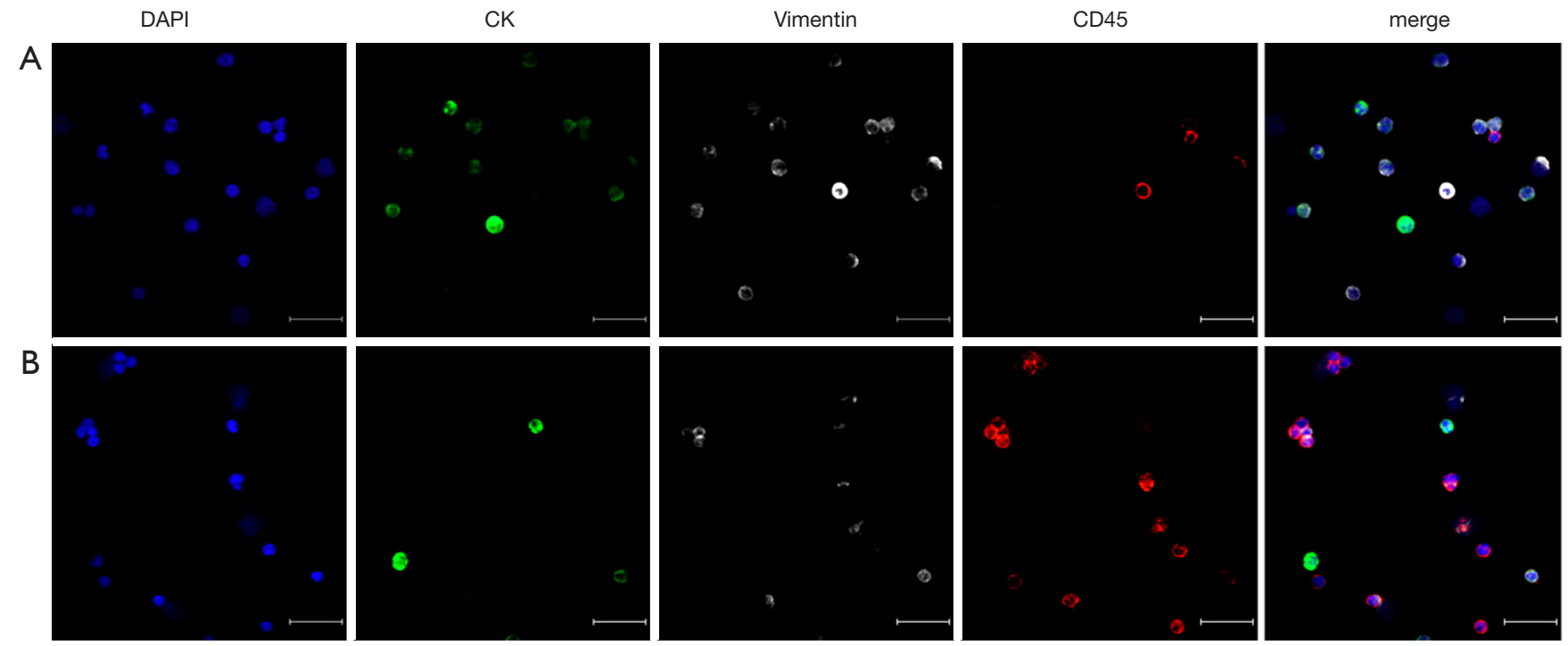

Figure S1 Multiple fluorescent images containing CTCs and residual white blood cells from the same patient. Images were from the portal blood sample (A) and the peripheral blood sample (B). DAPI, blue; CK, green; Vimentin, white and CD45, red. Scale bar: $30 \mu m$. CTCs, circulating tumor cells. 